\title{
Prompt photon production and photon-jet correlations at the LHC
}

\section{Michael Klasen, ${ }^{a}$ Christian Klein-Bösing ${ }^{b, c}$ and Hendrik Poppenborg ${ }^{b}$}

${ }^{a}$ Institut für Theoretische Physik, Westfälische Wilhelms-Universität Münster, Wilhelm-Klemm-Straße 9, D-48149 Münster, Germany

${ }^{b}$ Institut für Kernphysik, Westfälische Wilhelms-Universität Münster, Wilhelm-Klemm-Straße 9, D-48149 Münster, Germany

${ }^{c}$ ExtreMe Matter Institute EMMI, GSI Helmholtzzentrum für Schwerionenforschung, Planckstraße 1, D-64291 Darmstadt, Germany

E-mail: michael.klasen@uni-muenster.de,

c.klein-boesing@uni-muenster.de, hendrik.poppenborg@uni-muenster.de

ABSTRACT: Next-to-leading order predictions matched to parton showers are compared with recent ATLAS data on isolated photon production and CMS data on associated photon and jet production in $\mathrm{pp}$ and $\mathrm{pPb}$ collisions at different centre-of-mass energies of the LHC. We find good agreement and, as expected, considerably reduced scale uncertainties compared to previous theoretical calculations. Predictions are made for the ratio of inclusive photons over decay photons $R_{\gamma}$, an important quantity to evaluate the significance of additional photon sources, e.g. thermal radiation from a Quark-Gluon-Plasma, and for distributions in the parton momentum fraction in lead ions $x_{\mathrm{Pb}}^{\mathrm{obs}}$, that could be determined by ALICE, ATLAS, CMS and LHCb in ongoing analyses of photon+jet production in $\mathrm{pPb}$ collisions at $\sqrt{s_{N N}}=5.02 \mathrm{TeV}$. These data should have an important impact on the determination of nuclear effects such as shadowing at low $x$.

Keywords: Heavy Ion Phenomenology, NLO Computations

ARXIV EPRINT: 1709.04154 


\section{Contents}

1 Introduction 1

2 Prompt photon production with POWHEG 2

2.1 NLO calculation of prompt photon production and matching to PS 2

2.2 Sensitivity of the QED PS to the cutoff scale 4

3 Comparisons with current LHC data 5

3.1 Isolated photon production in pp collisions with ATLAS 5

$\begin{array}{lll}3.2 & \text { Photon-jet correlations in pp and } \mathrm{pPb} \text { collisions with CMS } & 7\end{array}$

4 Predictions for future LHC analyses and sensitivity to nuclear PDFs $\quad 10$

$\begin{array}{lll}\text { 4.1 Ratio of inclusive over decay photons with ALICE } & 11\end{array}$

4.2 Photon-jet production at high $p_{T}^{\gamma}$ in $\mathrm{pPb}$ collisions with ATLAS or CMS 13

$\begin{array}{lll}4.3 & \text { Photon-jet production at low } p_{T}^{\gamma} \text { in } \mathrm{pPb} \text { collisions with ALICE } & 14\end{array}$

4.4 Forward photon-jet production in p-Pb and $\mathrm{Pb}-\mathrm{p}$ collisions with $\mathrm{LHCb} \quad 15$

5 Conclusions and outlook $\quad 16$

\section{Introduction}

Photons play many important roles in high-energy collisions. They allow, e.g., to determine the strong coupling constant $\left(\alpha_{s}\right)$ [1] or the partonic structure of protons, photons [2] and nuclei $[3-5]$, and they can serve as a thermometer of the hot Quark-Gluon-Plasma (QGP) created in heavy-ion collisions [6, 7]. If produced in association with a hadronic jet, they allow to calibrate the transverse momentum of the latter at its creation and to deduce its subsequent hadronic energy loss or azimuthal decorrelation in the medium [8].

Theoretical predictions for final-state photon production in $\mathrm{pp}, \mathrm{pPb}$, and $\mathrm{PbPb}$ collisions have since long been available at leading order (LO) of perturbative QCD and have been implemented in parton shower (PS) Monte Carlo generators like PYTHIA 8 [9]. The latter also allow to model the (vacuum) fragmentation of final-state quarks and gluons into the observed hadrons and jets. Here, detailed information on the final state comes at the price of large theoretical uncertainties from variations of unphysical scales and other parameters. Nevertheless, Monte Carlo generators represent an indispensable tool in the experimental analyses such as those of the ALICE [10, 11], ATLAS [12, 13], CMS [14], and LHCb [15] experiments at the Large Hadron Collider (LHC) at CERN.

In contrast, inclusive next-to-leading order (NLO) QCD calculations [16, 17] depend on fewer tunable parameters and allow for a stabilisation of renormalisation and factorisation scale dependences among perturbative matrix elements and renormalisation-group 
improved initial-state parton density functions (PDFs) and final-state fragmentation functions (FFs). As a consequence, they have been widely applied to photon measurements at the Tevatron, most recently to single [18] and pairs of isolated photons [19], photon+jet [20], and (with shortcomings at high $p_{T}$ ) photon+heavy quark measurements [21, 22]. Unfortunately, PDFs and FFs are non-perturbative and must be fitted to experimental data [23]. Due to the lack of a new electron-positron collider, few improvements have been made on photon FFs over the last decades [24, 25]. NLO calculations for $Z \gamma+$ jet and $Z \gamma \gamma$ [29] have recently become available in MCFM, followed by predictions at next-to-next-to-leading order (NNLO) for diphoton [26, 27] and $Z_{\gamma}[28]$ production. The disadvantages of inclusive calculations lie in the limited multiplicity of and information on the produced final state and the consequently restricted number of kinematic observables.

Matching a NLO calculation with a PS Monte Carlo generator combines the advantages of both methods. Subtraction methods [30] like the one implemented in POWHEG [31] to avoid double counting of the soft and collinear regions are now well established. We have recently applied this method to prompt photon production and associated photonjet production in proton-proton ( $\mathrm{pp}$ ) collisions and compared our numerical results with PHENIX data from RHIC at BNL [32]. An important difference of NLO+PS calculations with respect to inclusive NLO calculations lies in the treatment of fragmentation contributions. It has, however, been demonstrated that in electron-positron collisions the NLO+PS approach also leads to a very good description of FF photons [33]. Multijet merging strategies have also recently been developed and applied in particular to diphoton production at NLO [34-36] and at NLO+PS [37].

It is the purpose of this paper to confront our NLO+PS calculations employing POWHEG+PYTHIA for the first time with LHC data. After a review of our theoretical method and a demonstration of the parameter dependence of LO+PS calculations in section 2, we perform these comparisons in section 3 first for isolated photon production, measured in pp collisions by ATLAS [12], then for associated photon+jet production, analysed in pp and $\mathrm{pPb}$ collisions by CMS [14]. Predictions for these experiments as well as for the ALICE and $\mathrm{LHCb}$ experiments are made in section 4 with a focus on the determination of nuclear PDFs in $\mathrm{pPb}$ collisions and on the reliable calculation of quantities relevant for the extraction of QGP properties. Our conclusions are presented in section 5.

\section{Prompt photon production with POWHEG}

In this section, we summarise our theoretical calculation of prompt photon production at NLO and the implementation of this calculation in POWHEG. Details can be found in our previous publication [32].

\subsection{NLO calculation of prompt photon production and matching to PS}

Direct photon production proceeds at LO through the tree-level processes $q \bar{q} \rightarrow \gamma g$ and $q g \rightarrow \gamma q$. In the POWHEG method [31], these processes must also be calculated with colour and spin correlations. We computed the traces of Dirac matrices with FormCalc 8.4 [38] and checked our results against the literature and Madgraph 5 [39]. 
The virtual corrections required the computation of the same processes at the one-loop level, the subsequent reduction of tensor loop integrals with Form [40], and the computation of the resulting scalar integrals with LoopTools 2.13 [41]. Renormalisation was performed in the $\overline{\mathrm{MS}}$ scheme, and the UV-finite results were checked against MG5_aMC@NLO [42]. For the real corrections, the tree-level processes with additional gluon radiation or gluon splitting into quark-antiquark pairs had to be computed. The new process $g g \rightarrow \gamma q \bar{q}$ plays a particularly important role at the LHC due to the high gluon luminosity at these collision energies. Final-state quark-antiquark pairs lead to different jet fragmentation, in particular in the QGP produced in heavy-ion collisions. Again, traces of Dirac matrices were computed with FormCalc 8.4 and the results checked with Madgraph 5. Infrared singularities were then removed with the dipole subtraction method [43]. QCD finiteness was checked against AutoDipole 1.2.3 [44], and the integrated QED dipole was shown to reproduce well the pointlike contribution to the photon fragmentation function. The total NLO calculation was finally shown to agree with JETPHOX [16, 17]. The latter, however, also includes fragmentation contributions at NLO and requires the convolution with a photon fragmentation function such as BFG II [23]. In our POWHEG approach, these contributions are taken into account at NLO by partonic scatterings supplemented with a QED PS. For our central numerical results, we identified the renormalisation and factorisation scales $\mu_{R}$ and $\mu_{F}$ with the transverse momentum of the photon or leading underlying parton. To estimate the theoretical uncertainties, the scales were then varied by relative factors of two, but not four.

NLO calculations usually increase and, more importantly, stabilise the LO cross section with respect to scale variations. However, they include only one additional parton and no hadronisation effects. Conversely, PS Monte Carlo generators generally have LO normalisation and large scale dependence, but include many additional partons and different hadronisation models. Matching NLO with PS combines the advantages of both approaches. In POWHEG, the overlap in the soft/collinear regions of real emission processes is automatically subtracted with the FKS method [30], so that only finite (i.e. UVrenormalised and IR-subtracted) loop and unsubtracted real emission amplitudes must be provided. The hardest radiation is generated first, only Monte Carlo events with positive weight are produced, and the NLO calculation can be combined with various PS algorithms. In our work we use PYTHIA 8.226 [9] with its $p_{T}$-ordered parton shower and string hadronisation model and allow unstable short-lived particles to decay. We have checked that multiple interactions and soft underlying events, while included in our calculations, have no significant effects. To account for different definitions of radiation transverse momentum in POWHEG BOX and PYTHIA, we use the class PowhegHooks and have PYTHIA evolve the shower starting from the kinematical limit rather than the scale passed by the POWHEG BOX. We then translate the $p_{T}$ of a generated radiation from the PYTHIA definition to the POWHEG BOX definition and veto radiation harder than the hard POWHEG scale. Following ref. [45], we employ different hard scales for the QED and for the QCD shower. In the nomenclature of ref. [46], we use the so-called NC-scheme and modify the POWHEG BOX to pass the scales of both the underlying Born event and the first radiation to PYTHIA. For completeness, we list non-standard POWHEG parameter settings and options in tables 1 and 2 . 


\begin{tabular}{|c|c|c|c|c|c|c|c|c|c|}
\hline ncall1 & itmx1 & ncall2 & itmx2 & foldcsi & foldy & nubound & iymax & doublefsr & iupperfsr \\
\hline 500000 & 5 & 500000 & 5 & 2 & 5 & 500000 & 5 & 1 & 1 \\
\hline
\end{tabular}

Table 1. List of POWHEG BOX parameters set to different values than those in the manual.

\begin{tabular}{|c|c|c|c|c|c|}
\hline veto & vetoCount & pThard & pTemt & pTdef & QEDveto \\
\hline 1 & 50 & 0 & 0 & 1 & 0 \\
\hline
\end{tabular}

Table 2. List of POWHEGHooks options.

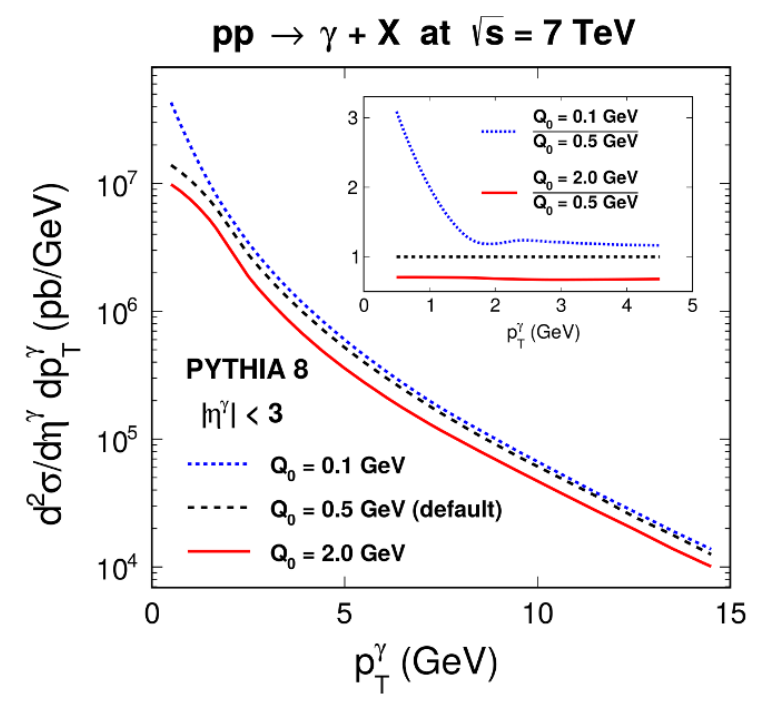

Figure 1. Transverse momentum dependence of inclusive photons produced in pp collisions at the LHC with a centre-of-mass energy of $\sqrt{s}=7 \mathrm{TeV}$ for different PS cut-off scales $Q_{0}$ in PYTHIA 8.

\subsection{Sensitivity of the QED PS to the cutoff scale}

An important difference between the inclusive fixed-order and the more differential PS calculations is the treatment of fragmentation contributions. At fixed order, infrared singularities are consistently factorised into non-perturbative fragmentation functions, whereas they are simply cut off in the PS by allowing it to evolve in virtuality, angle, or transverse momentum in each subsequent branching down to an arbitrary cut-off scale $Q_{0}^{2}$. The maximal allowed $Q^{2}$ is set by the hard scattering process.

The transverse momentum dependence of inclusive photons generated by the QED PS in the Monash 2013 tune of PYTHIA 8 is shown in figure 1. The photons are assumed to be produced in pp collisions at the LHC with a centre-of-mass energy of $\sqrt{s}=7 \mathrm{TeV}$ and detected in the CMS electromagnetic calorimeter with rapidity $\left|\eta^{\gamma}\right|<3$. No isolation criterion on the photons is applied. As in the Monash 2013 tune, the PDF fit NNPDF2.3QED LO with $\alpha_{s}\left(M_{Z}\right)=0.130$ is used. The dependence of the transverse momentum spectrum on the lower cut-off scale $Q_{0}$ can be deduced from the different curves and from the ratios of the variations $Q_{0}=0.1 \mathrm{GeV}$ (dotted blue) and $2 \mathrm{GeV}$ (full red) to the default choice $0.5 \mathrm{GeV}$ (dashed black) in the inset plot. Above transverse photon momenta of $p_{T}^{\gamma}=2 \mathrm{GeV}$, the results with $Q_{0}=0.1 \mathrm{GeV}$ and $Q_{0}=2 \mathrm{GeV}$ differ from those at $0.5 \mathrm{GeV}$ 


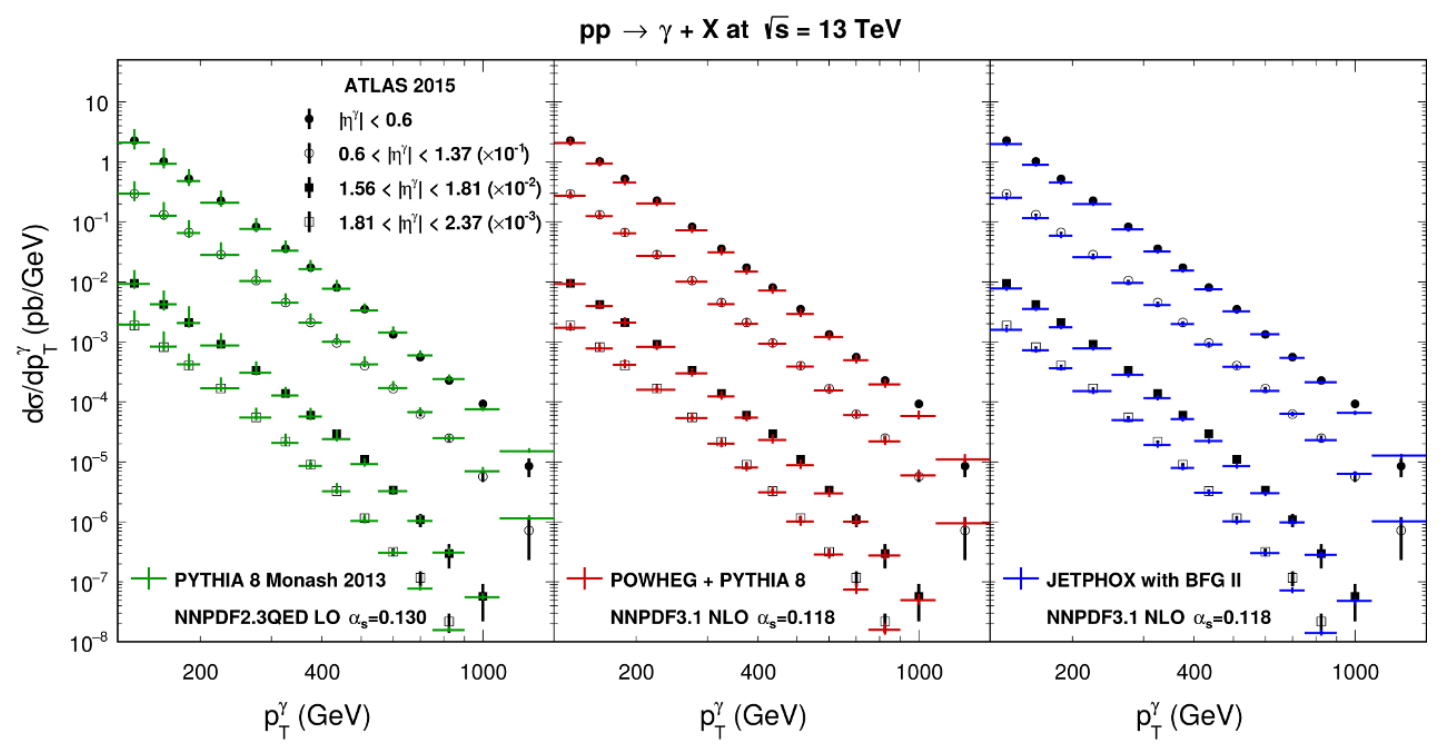

Figure 2. Transverse momentum distributions of isolated photons in pp collisions at the LHC with a centre-of-mass energy of $\sqrt{s}=13 \mathrm{TeV}$ in four different rapidity bins. ATLAS 2015 data are compared to predictions in LO with PYTHIA 8 (left), NLO with JETPHOX (right), and NLO+PS with POWHEG (centre).

only by a constant factor of $+10 \%$ and $-20 \%$, respectively. Towards low $p_{T}^{\gamma}$, the results with $Q_{0}=0.1 \mathrm{GeV}$ rise, however, much more steeply than those with $Q_{0}=0.5 \mathrm{GeV}$. These results demonstrate the strong dependence on and the need for tuning of the arbitrary cutoff $Q_{0}$ in a LO PS Monte Carlo generator. In contrast, the POWHEG approach guarantees for a correct cancellation of infrared singularities among NLO matrix elements and PS.

\section{Comparisons with current LHC data}

We now turn to comparisons of our NLO+PS predictions with POWHEG+PYTHIA with published LHC data. For comparison, we will also show calculations at LO+PS with PYTHIA 8 and at NLO with JETPHOX, since these were so far frequently used as theoretical baselines in the experimental publications.

\subsection{Isolated photon production in pp collisions with ATLAS}

The ATLAS collaboration has measured the production of inclusive photons in pp collisions at the LHC with a centre-of-mass energy of $\sqrt{s}=13 \mathrm{TeV}$ and an integrated luminosity of $3.2 \mathrm{fb}^{-1}$ accumulated in 2015 [12]. To avoid the large contribution of photons from decays of energetic neutral pions and $\eta$-mesons inside jets, the photons were isolated based on the amount of transverse energy $E_{T}^{\text {iso }}$ inside a cone of size $\Delta R=\sqrt{(\Delta \eta)^{2}+(\Delta \phi)^{2}}=0.4$ in the plane of rapidity $(\eta)$ and azimuthal angle $(\phi)$, excluding an area of size $\Delta \eta \times \Delta \phi=$ $0.125 \times 0.175$, around the photon candidate. Specifically, $E_{T}^{\text {iso }}$ was required to be lower than $4.8+4.2 \cdot 10^{-3} \cdot E_{T}^{\gamma}[\mathrm{GeV}]$.

In figure 2, we compare the transverse momentum distributions measured by ATLAS in four rapidity ranges to predictions in LO from the PYTHIA 8 PS Monte Carlo generator 


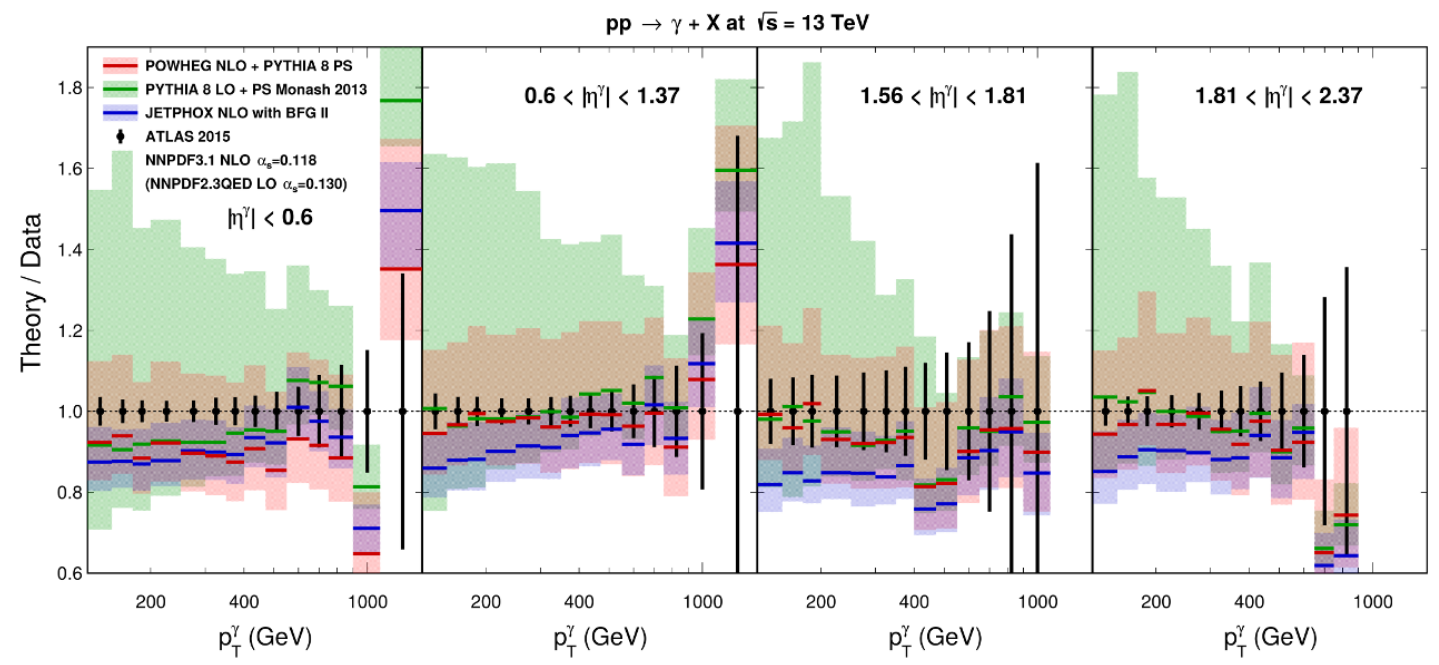

Figure 3. Same as figure 2, but with theory normalised to data and including scale uncertainties.

(green, left) [9], in NLO from the fixed-order program JETPHOX [16, 17] using the BFG II photon fragmentation function (blue, right) [23], and to our new NLO+PS predictions with POWHEG matched to PYTHIA 8 (red, centre) [32]. For the NLO and NLO+PS predictions, we employ the recent PDF set NNPDF3.1 NLO based on high-precision collider data [47]. For the LO predictions, we use instead the preceding set NNPDF2.3QED LO [48], which (while based on a similar theoretical approach) was in particular employed in the Monash 2013 tune of PYTHIA 8 [49]. For inclusive photon production, on a logarithmic scale, and thanks to the tuning of the Monte Carlo generator, all three predictions seem to agree very well with the data. Smaller discrepancies are, however, already visible in LO and NLO at high transverse momenta and/or very forward rapidities, while the NLO+PS predictions always agree with the data within the experimental error bars.

A more detailed analysis is performed in figure 3, where the theoretical predictions have been normalised to the central measurements. Theoretical uncertainties based on scale variations are shown in addition as shaded areas. As expected, the LO uncertainties are considerably larger than those at NLO and NLO+PS, which demonstrates that the agreement of the LO central values is rather accidental and depends on the tuning. The NLO JETPHOX predictions have a different shape in the second rapidity bin and globally underestimate the data even within error bars. This is even more the case for NNPDF2.3QED NLO PDFs (not shown, but known [50]). The results with MMHT2014, CT14 and NNPDF3.0 PDFs are very similar [12]. While the central POWHEG NLO+PS predictions still underestimate the data in the central rapidity bin, they generally agree best with the data and always within scale uncertainties. Since our NLO calculation of direct photon and purely partonic processes in POWHEG agrees with those in JETPHOX [32], this seems to indicate that the PS fragmentation in PYTHIA 8 describes the data slightly better than the BFG II fragmentation function. 


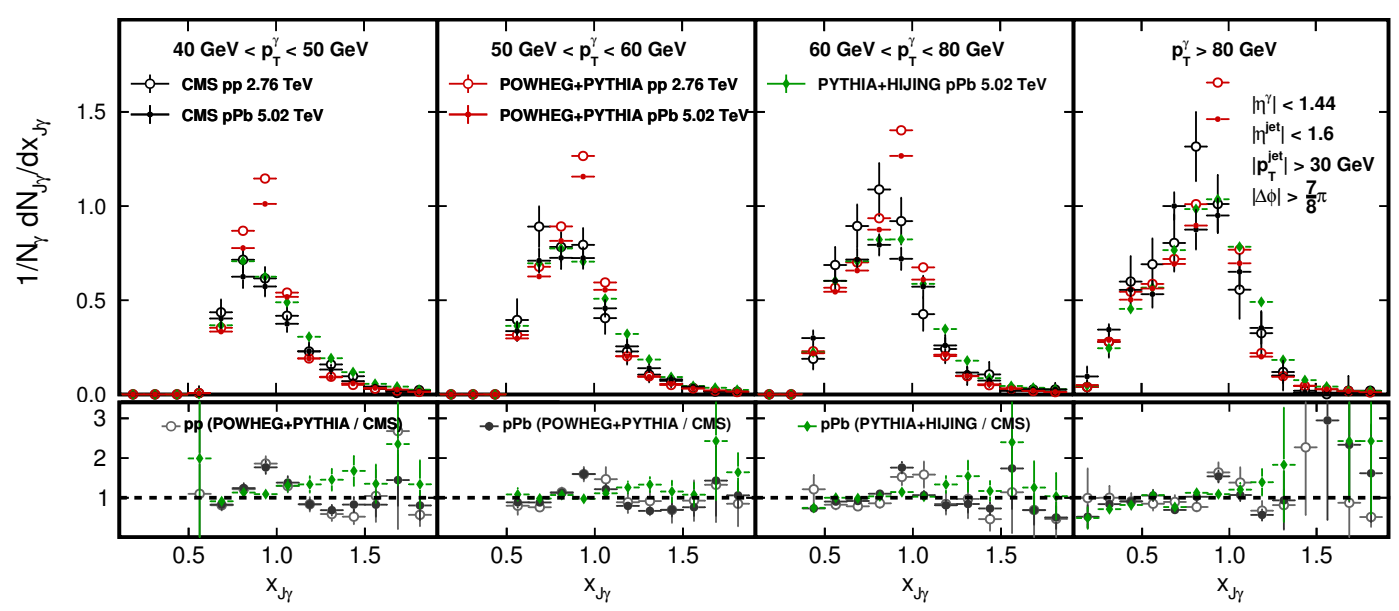

Figure 4. Distributions in the transverse momentum ratio of jets over photons in pp (open circles) and $\mathrm{pPb}$ (full circles) collisions at the LHC with a centre-of-mass energy per nucleon of $\sqrt{s_{N N}}=2.76$ and $5.02 \mathrm{TeV}$, respectively, in four different bins of photon transverse momentum. CMS 2013 data (black) are compared to predictions in LO with PYTHIA+HIJING (green) and NLO+PS with POWHEG+PYTHIA (red). The lower panels show the ratios of theory over data for pp (open circles) and $\mathrm{pPb}$ (full circles and green diamonds).

\subsection{Photon-jet correlations in $\mathrm{pp}$ and $\mathrm{pPb}$ collisions with $\mathrm{CMS}$}

Compared to inclusive photon production, photon-jet correlations are more sensitive to initial- and final-state QCD effects like PDFs, photon and jet fragmentation, and their modifications in nuclear collisions. They have therefore recently been measured by the CMS collaboration in pp and $\mathrm{PbPb}$ collisions at a nucleon-nucleon centre-of-mass energy of $\sqrt{s_{N N}}=2.76 \mathrm{TeV}$ and in $\mathrm{pPb}$ collisions at a nucleon-nucleon centre-of-mass energy of $\sqrt{s_{N N}}=5.02 \mathrm{TeV}$ [14]. During $2013(\mathrm{pp}), 2011(\mathrm{PbPb})$, and $2013(\mathrm{pPb})$, integrated luminosities of $5.3 \mathrm{pb}^{-1}, 150 \mu \mathrm{b}^{-1}$, and $30.4 \mathrm{nb}^{-1}$ were accumulated, respectively. The pp collisions are usually considered to be the (vacuum) baseline with equal photon and jet transverse momentum at LO, although this no longer holds at NLO and beyond (see below). "Hot" $\mathrm{PbPb}$ collisions are then studied to establish the creation of a QGP and to determine its properties, in particular through jet quenching (parton energy loss), while "cold" pPb collisions allow to check the absence of nuclear effects in the final state and to quantify the modifications of the PDFs in the initial state.

Similarly to the ATLAS analysis described above, CMS applied an isolation criterion to the photons, albeit with a fixed cut on the total energy $E^{\text {iso }}$ at $5 \mathrm{GeV}$. In addition, photons which matched a track from charged particles within $\left|\eta^{\gamma}-\eta^{\text {Track }}\right|<0.02$ and $\left|\phi^{\gamma}-\phi^{\text {Track }}\right|<0.15$ were discarded in order to remove contamination from electrons, and the remaining photons had to satisfy $p_{T}^{\gamma}>40 \mathrm{GeV}$ and $\left|\eta^{\gamma}\right|<1.44$. Jets were defined with an anti- $k_{T}$ cluster algorithm [51] and a distance parameter $R=0.3$ and had to satisfy $p_{T}^{\text {jet }}>30 \mathrm{GeV}$ and $\left|\eta^{\text {jet }}\right|<1.6$ to remain in the barrel calorimeter.

The distributions in figure 4 depend on the transverse momentum ratio of jets over photons $x_{\mathrm{J} \gamma}=p_{T}^{\mathrm{jet}} / p_{T}^{\gamma}$, which in LO would equal unity. If the photon is associated with several jets, each of them contributes in the histograms, but the main contribu- 


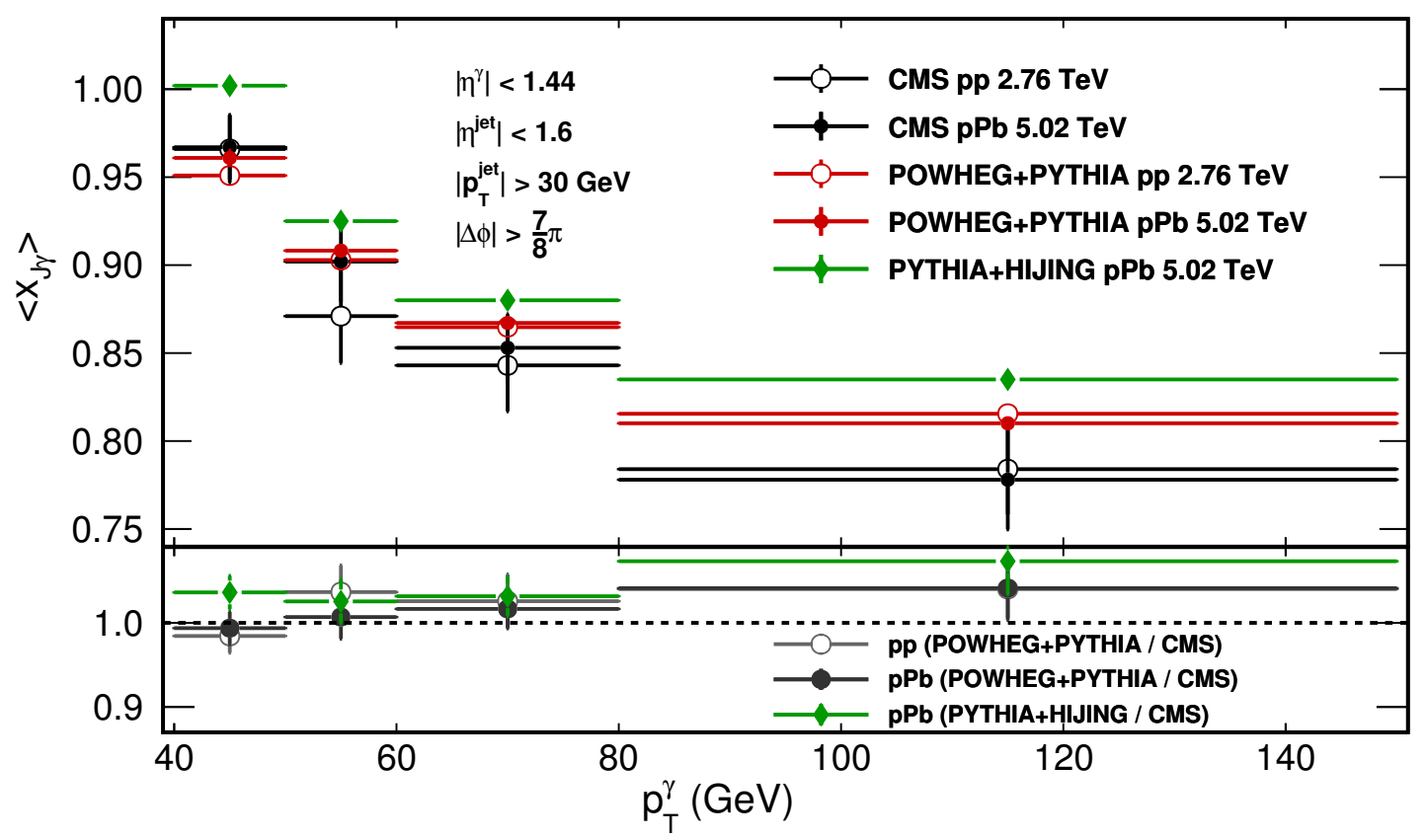

Figure 5. Average jet transverse momentum fraction as a function of photon transverse momentum. CMS 2013 data (black) are compared to predictions in LO with PYTHIA+HIJING (green) and NLO+PS with POWHEG+PYTHIA (red). The rightmost $p_{T}^{\gamma}$-bin refers to $p_{T}^{\gamma}>80 \mathrm{GeV}$ (no upper bound). The lower panel shows the ratio of theory over data for pp (open circles) and $\mathrm{pPb}$ (full circles and green diamonds), which coincide partially for the largest $p_{T}^{\gamma}$-bin.

tion comes from photon plus one jet events. The reason is that a cut on $\Delta \phi_{\mathrm{J} \gamma}>7 \pi / 8$ is applied to each of the jets to suppress contributions from background and additional jets. Since the pp (open circles) and $\mathrm{pPb}$ (full circles) data are consistent with each other and the PYTHIA+HIJING [52] Monte Carlo simulation, nuclear effects and centreof-mass energy dependence must be subdominant. However, the jets become softer at larger photon transverse momenta relative to the photon. Our NLO+PS calculations with POWHEG+PYTHIA describe the data well. They have a slightly sharper peak at somewhat larger values of $x_{\mathrm{J} \gamma}$, since they have not been smeared with the resolution of the CMS detector.

The average values of $x_{\mathrm{J} \gamma}$ are plotted in figure 5 as a function of the photon transverse momentum. Again, there are no significant differences between the pp and $\mathrm{pPb}$ data, supporting the interpretation that no QGP is created and rescattering effects in the medium can thus not occur. This is in contrast to a more recent measurement by CMS in $\mathrm{PbPb}$ collsions at $\sqrt{s_{N N}}=5.02 \mathrm{TeV}$, which is considerably lower than the pp baseline even after accounting for different jet resolutions [53]. At higher $p_{T}^{\gamma}$, several jets can jointly balance the transverse momentum of the photon, e.g. in a "Mercedes star" configuration, which leads to the observed falling distribution in $\left\langle x_{\mathrm{J} \gamma}\right\rangle$.

Possible medium effects of the back-to-back photon and recoiling jet alignment can also be studied by comparing relative azimuthal angle $\left(\Delta \phi_{J \gamma}\right)$ distributions in proton and heavy-ion collisions. Figure 6 displays these distributions in $\mathrm{pp}$ and $\mathrm{pPb}$ collisions in 


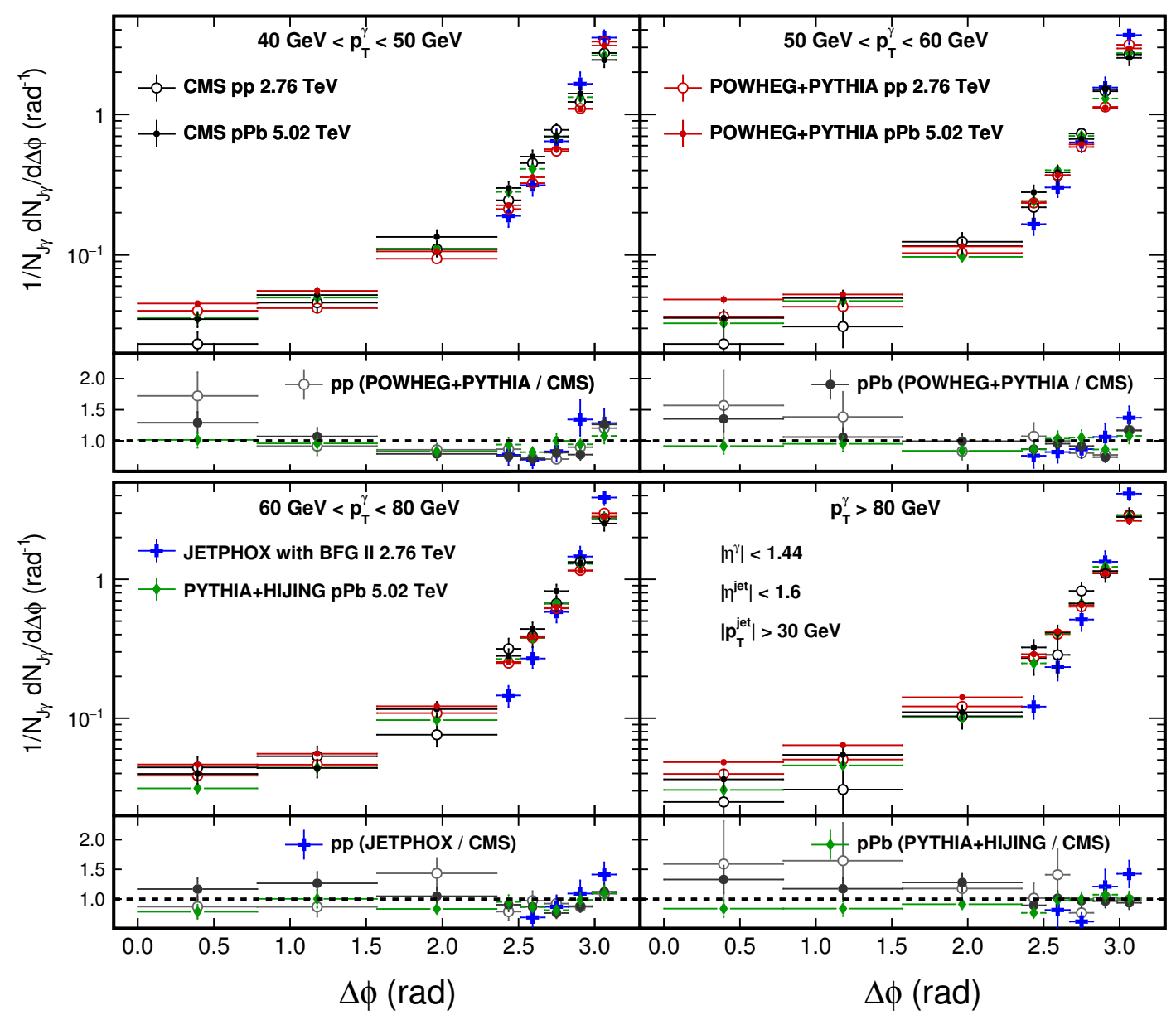

Figure 6. Relative azimuthal angle distributions of jets and photons in pp (open circles) and $\mathrm{pPb}$ (full circles) collisions at the LHC with a centre-of-mass energy per nucleon of $\sqrt{s_{N N}}=2.76$ and $5.02 \mathrm{TeV}$, respectively, in four different bins of photon transverse momentum. CMS 2013 data (black) are compared to predictions in LO with PYTHIA+HIJING (green), NLO with JETPHOX (blue), and NLO+PS with POWHEG+PYTHIA (red). The lower panels show the ratios of theory over data for pp (open circles and blue crosses) and $\mathrm{pPb}$ (full circles and green diamonds).

the same four bins of photon transverse momentum used above. Again, no significant cold nuclear effects or centre-of-mass energy dependences are visible in the data. The LO predictions with PYTHIA+HIJING agree well with the measurements, as do those at NLO+PS with POWHEG+PYTHIA. Note that the near-side region at small $\Delta \phi_{J \gamma}$ is quite sensitive to the experimental conditions on the photon isolation. At larger $p_{T}^{\gamma}$, the distributions become slightly flatter, which can again be traced to multi-jet configurations (note that here no cut on $\Delta \phi_{\mathrm{J} \gamma}>7 \pi / 8$ is applied). Azimuthal angle distributions can also be computed with JETPHOX, but they do not describe the data below $\Delta \phi_{J \gamma}=2 \pi / 3$, as the maximum number of jets is limited to two at NLO.

Not all photon events in the CMS measurement include a jet that passes the cut on $p_{T}^{\text {jet }}>30 \mathrm{GeV}$. The fraction of isolated photons that are associated to a jet is therefore less than unity and shown in figure 7 as a function of the photon transverse momentum. Here, 


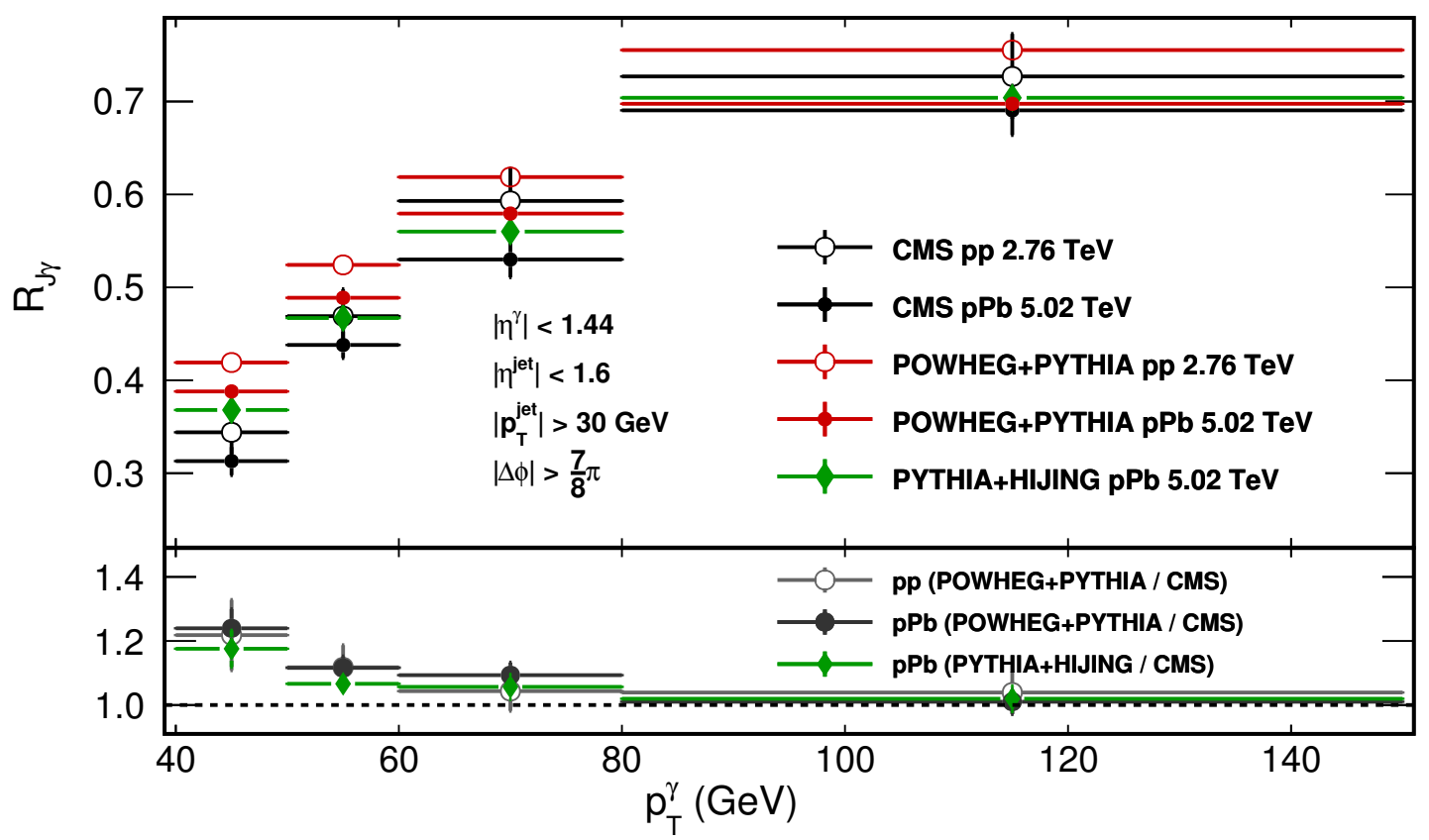

Figure 7. Fraction of photons associated to a jet with $p_{T}^{\text {jet }}>30 \mathrm{GeV}$ as a function of photon transverse momentum in pp (open circles) and $\mathrm{pPb}$ (full circles) collisions at the LHC with a centre-of-mass energy per nucleon of $\sqrt{s_{N N}}=2.76$ and $5.02 \mathrm{TeV}$, respectively. CMS 2013 data (black) are compared to predictions in LO with PYTHIA+HIJING (green) and NLO+PS with POWHEG+PYTHIA (red). The lower panel shows the ratio of theory over data for pp (open circles) and $\mathrm{pPb}$ (full circles and green diamonds).

again a cut on $\Delta \phi_{\mathrm{J} \gamma}>7 \pi / 8$ is applied to suppress contributions from background and additional jets. Within the experimental uncertainties, neither nuclear nor centre-of-mass energy dependences are observed, although $\mathrm{pPb}$ collisions globally seem to have a smaller $R_{J \gamma}$. The $\mathrm{pPb}$ cross section is affected by nuclear shadowing (in particular of the gluons) at $x_{T}^{\gamma} \geq 2 \times 40 \mathrm{GeV} / 5020 \mathrm{GeV} \geq 0.016$. This not only reduces the inclusive photon cross section (which would not affect $R_{J \gamma}$, since most photons recoil against jets), but fewer initial gluons also lead to fewer quark jets, which would pass the cut on $p_{T}^{\text {jet }}>30 \mathrm{GeV}$ more easily than gluon jets (which does affect $R_{J \gamma}$ ). This (statistically not significant) observation is reproduced by our NLO POWHEG+PYTHIA calculations, but they slightly overestimate both the pp and $\mathrm{pPb}$ data. Note that the ATLAS collaboration has recently also presented a measurement of isolated-photon and jet production, but only in pp collisions at $\sqrt{s}=$ $13 \mathrm{TeV}$ [54].

\section{Predictions for future LHC analyses and sensitivity to nuclear PDFs}

In this section we make NLO+PS predictions for future LHC analyses and compare them to those at LO+PS and NLO. First, we study the impact of higher-order corrections on the ratio of inclusive over decay photons. This quantity has been shown by ALICE to be of crucial importance in the determination of the effective temperature of the QGP. Second, we study the sensitivity of photon+ jet measurements in $\mathrm{pPb}$ collisions with ALICE, ATLAS, 
CMS and LHCb to nuclear PDFs. In particular, we show that distributions in the observed momentum fraction of the parton in the lead ion $x_{\mathrm{Pb}}^{\mathrm{obs}}$ are sensitive to nuclear effects like shadowing at low $x$. Related variables have previously been used successfully for proton and photon PDF determinations at the HERA collider at DESY.

\subsection{Ratio of inclusive over decay photons with ALICE}

Inclusive photons represent an essential probe of the medium produced in central heavyion collisions, since the thermal contribution to the exponentially falling spectrum at low transverse momenta can be related to an effective temperature of the QGP. In contrast, the hard perturbative contributions, e.g. from QCD Compton and photon-gluon fusion processes, lead to a power-law behaviour in $p_{T}^{\gamma}$. The corresponding measurements in pp collisions serve as a (vacuum) baseline and have been performed with 2010 and 2011 data by the ALICE collaboration at centre-of-mass energies of $0.9,2.76$ and $7 \mathrm{TeV}$ [10]. To remain sensitive to the thermal radiation, the photons must, however, not be isolated, leading to the complication that the spectra are dominated by contributions from neutral pion, $\eta$-, and $\omega$-meson decays.

Decay photon contributions were subtracted only in a subsequent ALICE measurement of $\mathrm{PbPb}$ collisions at $\sqrt{s_{N N}}=2.76 \mathrm{TeV}$ [11]. This measurement was reanalysed in NLO and including theoretical uncertainties in ref. [7]. The crucial quantity in the subtraction procedure was the $p_{T}^{\gamma}$-dependent double ratio

$$
R_{\gamma}=\frac{\gamma_{\text {incl. }}}{\pi_{\text {param. }}^{0}} / \frac{\gamma_{\text {decay }}}{\pi_{\text {param. }}^{0}},
$$

where $\gamma_{\text {incl. }}$ is the measured inclusive photon spectrum, $\pi_{\text {param. }}^{0}$ is a parameterisation of the measured $\pi^{0}$ spectrum, and $\gamma_{\text {decay }}$ is the calculated decay photon spectrum. It has the advantage that some of the largest systematic uncertainties cancel. The quantity $R_{\gamma}$ allowed to measure prompt (i.e. non-decay) photons with $0.9 \mathrm{GeV}<p_{T}^{\gamma}<14 \mathrm{GeV}$ and $\left|\eta^{\gamma}\right|<0.9$ in $\mathrm{PbPb}$ collisions through $\gamma_{\text {prompt }}=\left(1-1 / R_{\gamma}\right) \gamma_{\text {incl. }}$ for different centralities and to extract an effective temperature of the QGP in the most central collisions. A similar measurement is currently in progress for $\mathrm{PbPb}$ collisions at $\sqrt{s_{N N}}=5.02 \mathrm{TeV}$.

Since $R_{\gamma}$ relied on LO Monte Carlo simulations of the decay photon spectrum with PYTHIA, it is important to quantify the impact of higher-order effects on this observable. In figure 8 we show simulations of the (simplified) theoretical ratio

$$
R_{\gamma}=\frac{\gamma_{\text {incl. }}}{\gamma_{\text {decay }}}=1+\frac{\gamma_{\text {prompt }}}{\gamma_{\text {decay }}}
$$

for the current pp run at $\sqrt{s}=13 \mathrm{TeV}$. This quantity compared to data will help to constrain additional sources of low- $p_{T}^{\gamma}$ photons at the highest LHC energy, allowing to validate the thermal origin of photons measured in $\mathrm{PbPb}$ collisions. In figure 8 , the prompt photon spectrum is calculated in LO with PYTHIA (green), NLO with JETPHOX and BFG II photon fragmentation functions (blue), and NLO+PS with POWHEG+PYTHIA (red, full circles), respectively. In contrast, the decay photon spectrum is always calculated in LO with the Monash 2013 tune of PYTHIA 8, which is known to describe the pion 


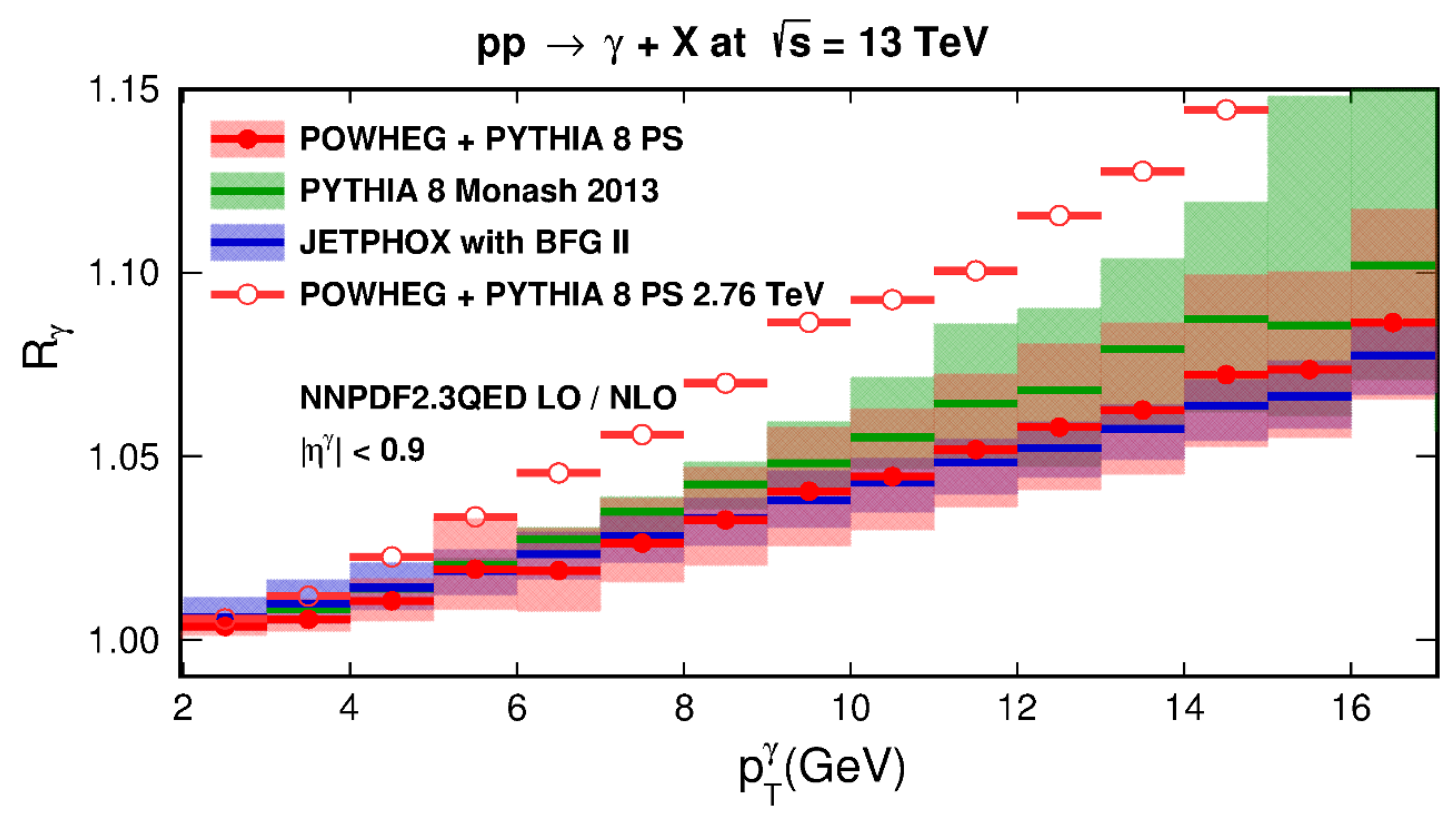

Figure 8. Ratio of inclusive over decay photon production in pp collisions at the LHC with a centre-of-mass energy of $\sqrt{s}=13 \mathrm{TeV}$. LO predictions with PYTHIA (green) are compared to those at NLO with JETPHOX (blue) and NLO+PS with POWHEG+PYTHIA (red, full circles). The latter are also shown at $\sqrt{s}=2.76 \mathrm{TeV}$ (red, open circles).

data very well, and does not contribute to the theoretical uncertainty. Once measured, the decay contribution should be replaced by the experimental values. For better comparability, NNPDF2.3QED PDFs are used in all calculations, since initial-state PDF effects are expected to cancel to a large extent. As expected, the scale uncertainties (shaded bands) are considerably larger at LO than at NLO and NLO+PS. The smaller scale uncertainty in NLO compared to NLO+PS is due to an additional cancellation of factorisation scale dependence in the fragmentation contribution. For $p_{T}^{\gamma}>7 \mathrm{GeV}$, the central values of $R_{\gamma}$ at LO are larger than those at NLO and NLO+PS and even fall outside the error band of the former. In contrast, the central values at NLO and NLO+PS agree very well and should therefore be employed in future experimental measurements.

For illustration we also show in figure 8 the POWHEG+PYTHIA results for pp collisions at $\sqrt{s}=2.76 \mathrm{TeV}$ (red, open circles). The direct photon signal is much more significant at lower $\sqrt{s}$ mainly due to the steeper spectra and larger probed $x$-values. Its contribution relative to the one by decay photons $\left(R_{\gamma}-1\right)$ changes for typical LHC energies from $13 \mathrm{TeV}$ to $2.76 \mathrm{TeV}$ by about a factor of two. A similar energy dependence is expected for the direct photon signal in $\mathrm{PbPb}$ collisions, since neither $T_{A A}$ nor the suppression of the hadronic decay background vary strongly with $\sqrt{s}$. The suppression of the decay background due to partonic energy loss in $\mathrm{PbPb}$ collisions leads in general to $R_{\gamma}$ values larger by factors of five to seven compared to pp collisions. 


\subsection{Photon-jet production at high $p_{T}^{\gamma}$ in $\mathrm{pPb}$ collisions with ATLAS or CMS}

In the remaining three subsections, we make predictions for associated photon and jet production in proton-lead collisions at a centre-of-mass energy per nucleon of $\sqrt{s_{N N}}=$ $5.02 \mathrm{TeV}$. Our focus will be on the potential of the four major LHC experiments ALICE, ATLAS, CMS and LHCb to improve on current constraints of nuclear PDFs with measurements of this reaction. In particular, we propose to analyse the experimental data in terms of distributions in the observed parton momentum fraction of the lead ion, defined as

$$
x_{\mathrm{Pb}}^{\mathrm{obs}}:=\frac{p_{T}^{\gamma} e^{-\eta^{\gamma}}+p_{T}^{\text {jet }} e^{-\eta^{\text {jet }}}}{2 E_{\mathrm{Pb}}},
$$

so that regions of nuclear shadowing and antishadowing, EMC effect and Fermi motion can directly be located in increasing regions of $x$. Related variables have been used successfully at DESY HERA for determinations of proton and photon PDFs [2].

We base our predictions on the current status of nuclear PDF determinations from deep-inelastic scattering (DIS) and Drell-Yan (DY) measurements and use for our central estimates the recent fit nCTEQ15(np) [55]. It is based on proton PDFs similar to CTEQ6.1 [56], but with minimal influence from nuclear data. For comparison, we will also show nCTEQ15 estimates with additional constraints from inclusive pion data taken by the PHENIX and STAR experiments at RHIC as well as estimates with the latest EPPS16 nuclear PDF fit [57] that already includes LHC data from ATLAS and CMS on electroweak gauge boson and dijet production in pPb collisions. The nCTEQ and EPPS16 fits come with 32 and 40 error PDFs, respectively, which we will show as shaded bands. We will, however, not vary the underyling proton PDF, assuming that it can be sufficiently constrained from pp collisions by the same experiments. Instead, we will show for comparison the pp baseline at $\sqrt{s}=2.76 \mathrm{TeV}$ with theoretical uncertainties obtained by scale variations of relative factors of two, but not four.

In this subsection, we concentrate on the ATLAS and CMS experimental acceptances. In particular, we require the photons to be isolated with $E^{\text {iso }}<5 \mathrm{GeV}$ inside a cone of size $\Delta R=0.4$, to have relatively high transverse momenta of $p_{T}^{\gamma}>40 \mathrm{GeV}$, and to remain in the CMS barrel electromagnetic calorimeter with $\left|\eta^{\gamma}\right|<1$.44. Jets, defined by the anti- $k_{T}$ cluster algorithm with distance parameter $R=0.3$, have to satisfy $p_{T}^{\text {jet }}>30 \mathrm{GeV}$ and remain in the CMS hadronic barrel calorimeter with $\left|\eta^{\text {jet }}\right|<1.6$. These cuts correspond to those used in section 3.2 [14]. The ATLAS acceptances with $\left|\eta^{\gamma}\right|<1.475$ and $\left|\eta^{\text {jet }}\right|<1.7$ are very similar [54]. The results of this proposed analysis are shown in figure 9 . The observable region of the parton momentum fraction in the lead ion ranges from 0.005 to 0.25 . The cross sections at $\sqrt{s}=2.76 \mathrm{TeV}$ are lower than those at $5.02 \mathrm{TeV}$ by up to a factor of five at low $x$. At NLO+PS, the scale uncertainties in pp collisions (grey) are still sizeable, so that they should be eliminated - like the proton PDF uncertainties (not shown) - by a corresponding baseline measurement or by taking ratios of nuclear and bare proton cross sections. The shadowing region below $x \sim 0.02$ is not well constrained by DIS and DY data (red) and exhibits uncertainties of up to a factor of two. The situation improves considerably after inclusion of hadron collider data from RHIC (green) or LHC (blue), which can also be expected from the proposed inclusive photon measurements. 


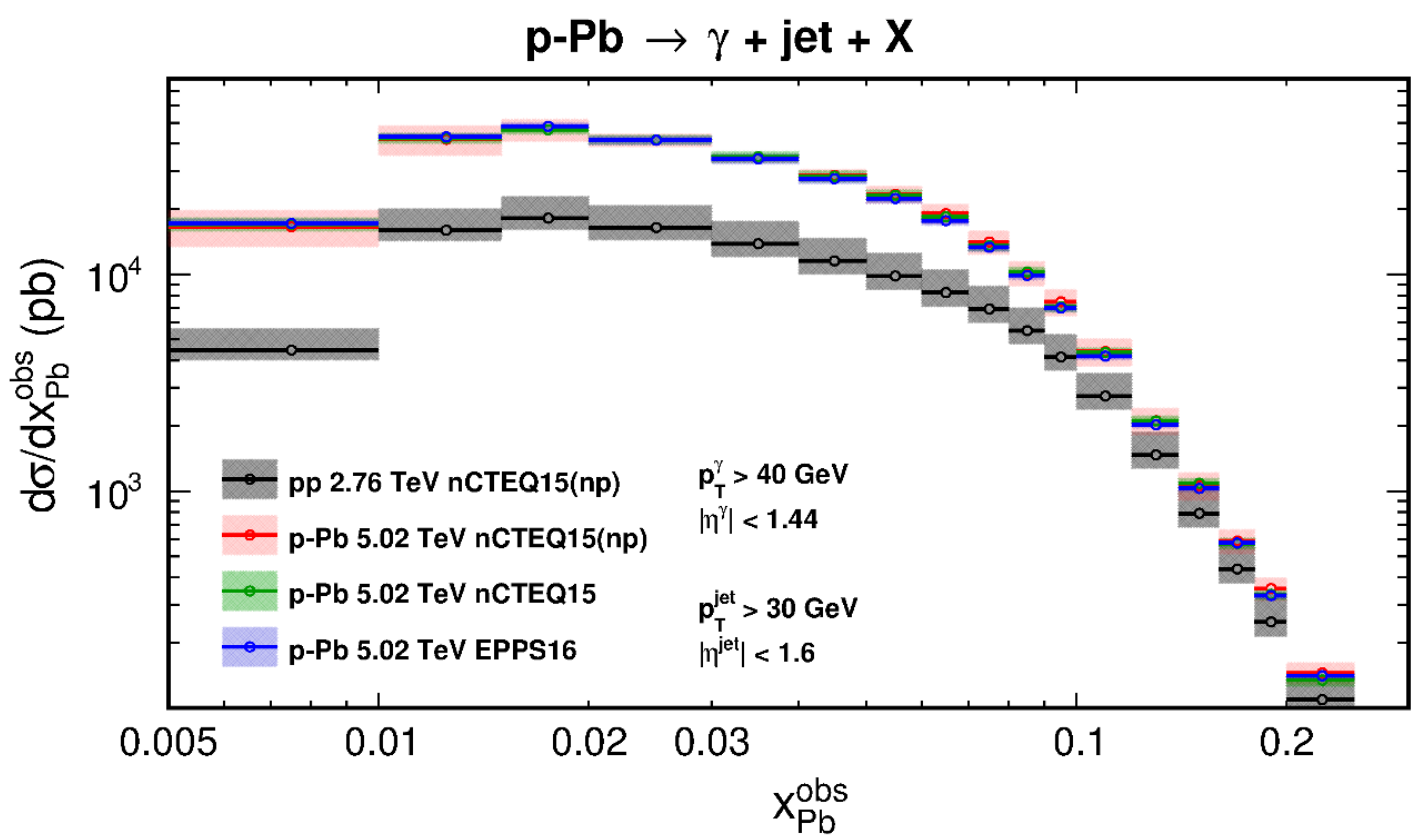

Figure 9. Nuclear PDF sensitivity of central high- $p_{T}^{\gamma}$ photon-jet measurements with ATLAS or CMS in $\mathrm{pPb}$ collisions at the LHC with a nucleon-nucleon centre-of-mass energy of $\sqrt{s_{N N}}=$ 5.02 TeV. Predictions with nCTEQ15(np) PDFs (red) are compared to those with nCTEQ15 including pion data from RHIC (green) and EPSS16 including weak boson and jet data from LHC (blue). The nCTEQ15 pp baseline is also shown (grey).

Similar improvements are observed in the antishadowing region above $x \sim 0.05$. In the intermediate region, nuclear effects are known to be small.

Increasing the photon acceptance to the CMS electromagnetic endcap calorimeter $\left|\eta^{\gamma}\right|<2.5$, but excluding the transition region of $1.44<|\eta|<1.57$, and the jet acceptance to $p_{T}^{\text {jet }}>25 \mathrm{GeV}$ and $\left|\eta^{\text {jet }}\right|<4.7$ as in ref. [58] leads to an extension of the observable $x$-range in the lead ion down to 0.001, as shown in figure 10 . Here, jets are in addition required to have a distance $\Delta R_{J \gamma}>1.0$ from the photon. As expected, the uncertainties from nuclear effects increase below $x$-values of 0.005 . This holds now not only for the fit of DIS and DY data only (red), but also for the fits that include RHIC (green) or LHC (blue) data, demonstrating the potential of inclusive photon and jet measurements to constrain the nuclear PDFs even further. The central fits of nCTEQ15(np), nCTEQ15 and EPPS16 are now also clearly distinguishable, the RHIC data favouring more, the LHC data less nuclear shadowing than the DIS and DY data alone.

\subsection{Photon-jet production at low $p_{T}^{\gamma}$ in $\mathrm{pPb}$ collisions with ALICE}

As discussed in section 4.1, the region of low transverse momenta gives access to the thermal photon spectrum produced in $\mathrm{PbPb}$ collisions. In addition, it should allow to probe even deeper into the low- $x$ shadowing region in $\mathrm{pPb}$ collisions. The low- $p_{T}$ region is well instrumented in the ALICE detector, so that in this subsection we apply the corresponding cuts on photons, isolated in a cone of size $R<0.4$ with $E_{T}^{\text {iso }}<2 \mathrm{GeV}[59]$, of $p_{T}^{\gamma}>1 \mathrm{GeV}$ 


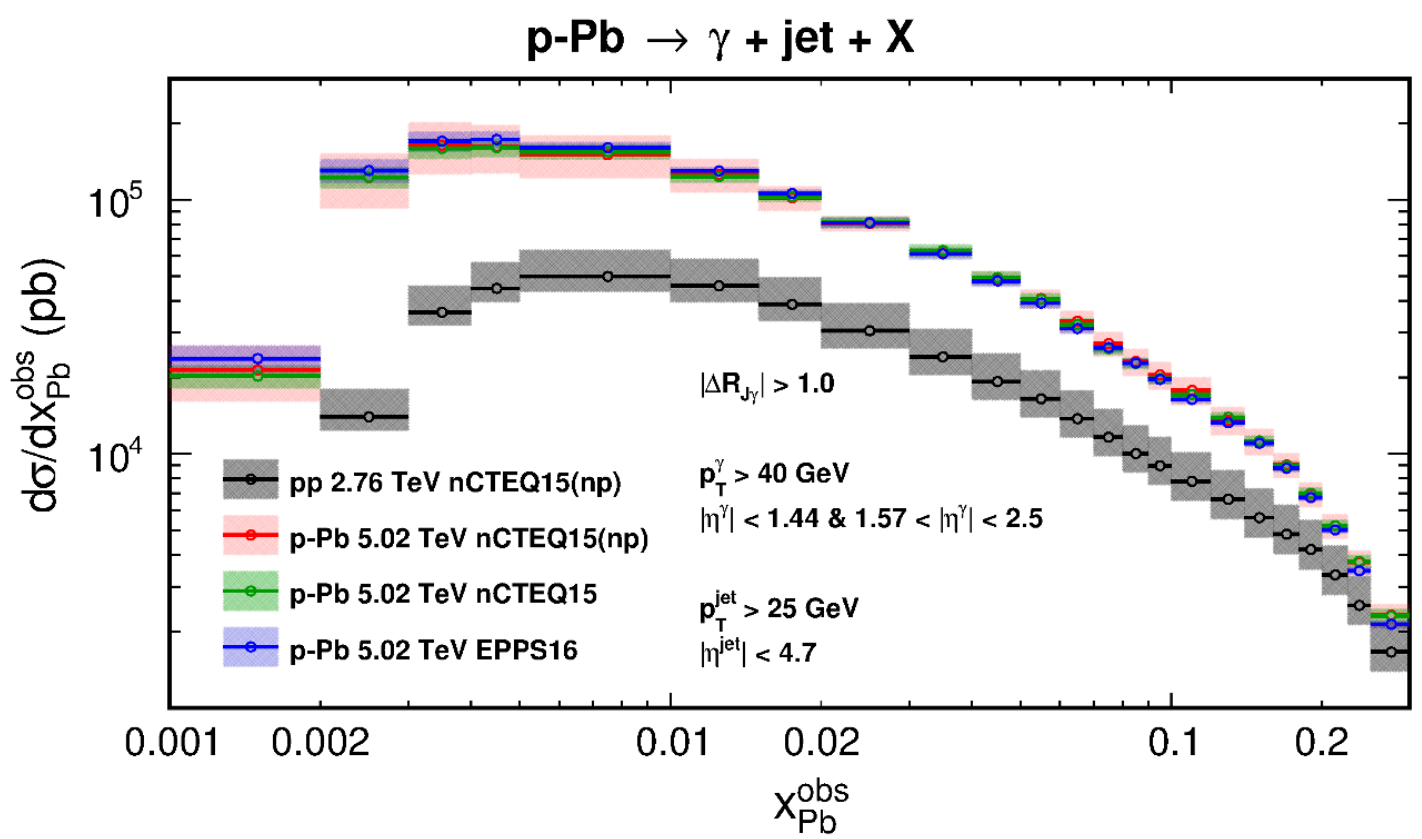

Figure 10. Same as figure 9, but with larger acceptances in photon and jet rapidity, using endcap calorimeters, and lower threshold on the jet transverse momentum.

and $\left|\eta^{\gamma}\right|<0.9$ [11] and on jets, defined by the anti- $k_{T}$ algorithm with $R=0.4$, of $p_{T}^{\text {jet }} \in$ $[20 ; 120] \mathrm{GeV}$ and $\left|\eta^{\text {jet }}\right|<0.5[60,61]$.

Figure 11 shows that this ALICE acceptance does not lead to lower observable values of $x$, but only to a similar $x$-range as described in the previous subsection, section 4.2, for central high- $p_{T}^{\gamma}$ analyses with ATLAS and CMS, contrary to what one might expect for lower $p_{T}^{\gamma}$. The reason is that the ALICE acceptance in rapidity is considerably more central than the one of ATLAS or CMS. It would therefore be benefitial if one could also use the ALICE Photon Multiplicity Detector (PMD) situated at forward rapidities of $2.3<\eta^{\gamma}<3.9[10]$.

\subsection{Forward photon-jet production in $\mathrm{p}-\mathrm{Pb}$ and $\mathrm{Pb}-\mathrm{p}$ collisions with $\mathrm{LHCb}$}

Although the main focus of the fourth LHC experiment LHCb is on precisision measurements of rare $B$ decays, the very forward instrumentation of the LHCb detector should also permit interesting measurements of other observables, that are complementary to the three other LHC experiments. Indeed, inclusive photons have been measured in radiative $B_{s}^{0}$ decays, even with polarisation, with $p_{T}^{\gamma}>3 \mathrm{GeV}$ and $\eta^{\gamma} \in[2 ; 5]$ [15]. For comparability with the previous subsection, we employ in addition the same photon isolation criterion as used by the ALICE collaboration [59]. Jets, defined by the anti- $k_{T}$ algorithm with $R=0.5$, have been measured by LHCb (in association with forward electroweak bosons) in the region $p_{T}^{\text {jet }} \in[20 ; 100] \mathrm{GeV}$ and $\eta^{\text {jet }} \in[2.2 ; 4.2][62]$.

We have employed these acceptances to $\mathrm{p}-\mathrm{Pb}$ collisions with the proton beam going in the positive $z$-axis direction. The lead ion is therefore always probed in the very small- $x$ region between $10^{-4}$ and $2 \times 10^{-3}$, as one can see in figure 12 . At $x \sim 10^{-4}$, the theoretical 


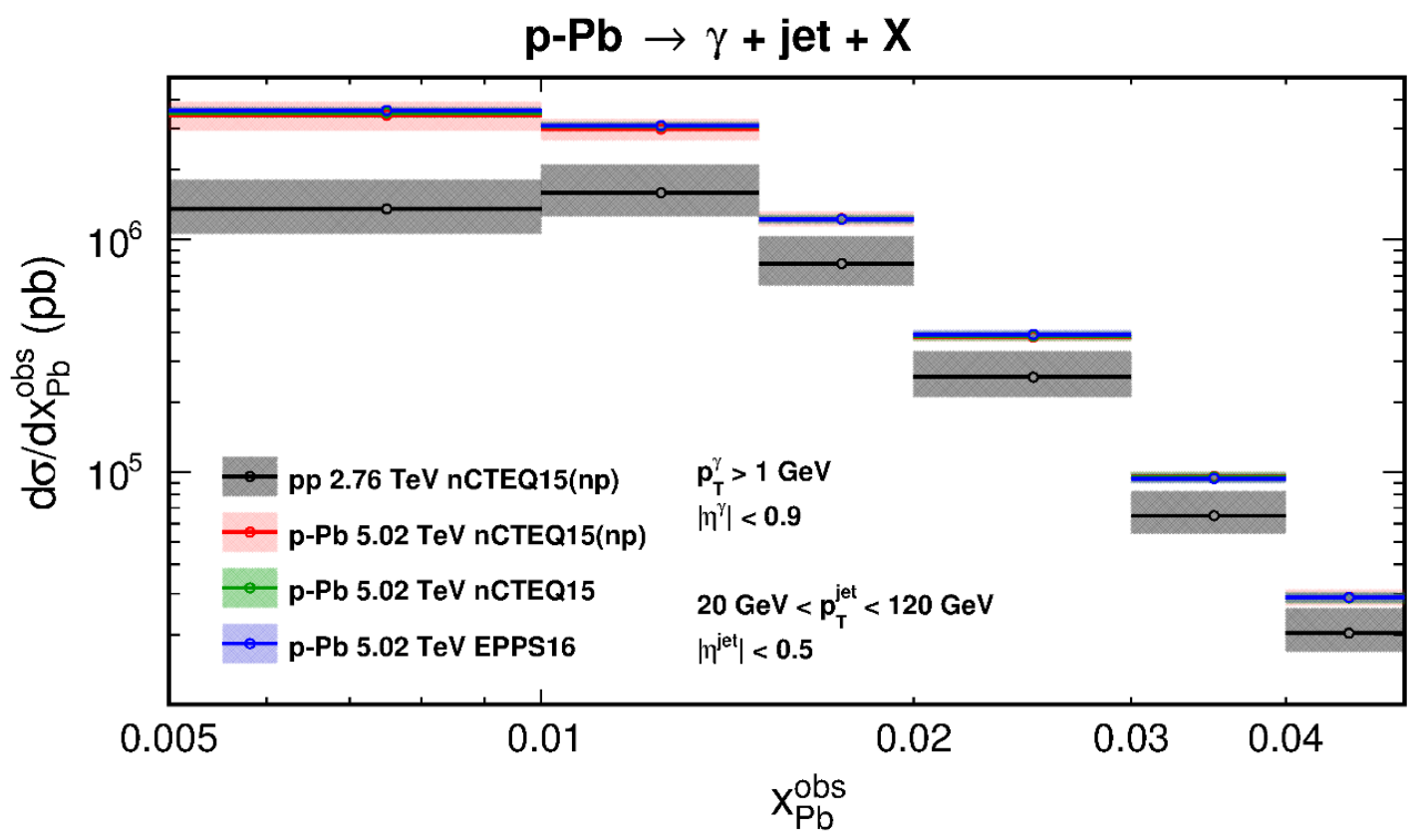

Figure 11. Nuclear PDF sensitivity of central low- $p_{T}^{\gamma}$ photon-jet measurements with ALICE in $\mathrm{pPb}$ collisions at the LHC with a nucleon-nucleon centre-of-mass energy of $\sqrt{s_{N N}}=5.02 \mathrm{TeV}$. Predictions with nCTEQ15(np) PDFs (red) are compared to those with nCTEQ15 including pion data from RHIC (green) and EPSS16 including weak boson and jet data from LHC (blue). The nCTEQ15 pp baseline is also shown (grey).

nuclear PDF uncertainty amounts to almost a factor of two for nCTEQ15(np) and 50\% for nCTEQ15 or EPPS16. Interestingly, both RHIC and LHC data now push the central predictions above those from DIS and DY data, i.e. towards less nuclear shadowing.

When the lead ion is going in the positive $z$-axis direction, it is mostly probed at large $x$, i.e. in the region of the EMC effect, as one can see in figure 13. This region is fairly well constrained by DIS and DY data alone, since the data from the EMC DIS experiment were heavily used in the nuclear PDF fits. So at large $x$, the uncertainty bands overlap quite well and shrink only moderately from nCTEQ15(np) to nCTEQ and EPPS16. Consequently, measurements in this "control" region could be used to fix the normalisation, i.e. the choices of scales or proton PDFs.

\section{Conclusions and outlook}

Prompt photons have many important applications in high-energy collisions. They range from determinations of the strong coupling constant, proton and nuclear PDFs to those of the properties of the QGP. Our recent calculation of prompt photon and photon+jet production at NLO+PS with POWHEG+PYTHIA combines the reliability of NLO calculations with detailed information on the final state as required for realistic phenomenological analyses.

In this paper, we have performed a first comparison of these calculations with LHC data, in particular of isolated photon production as measured by ATLAS and of photons 


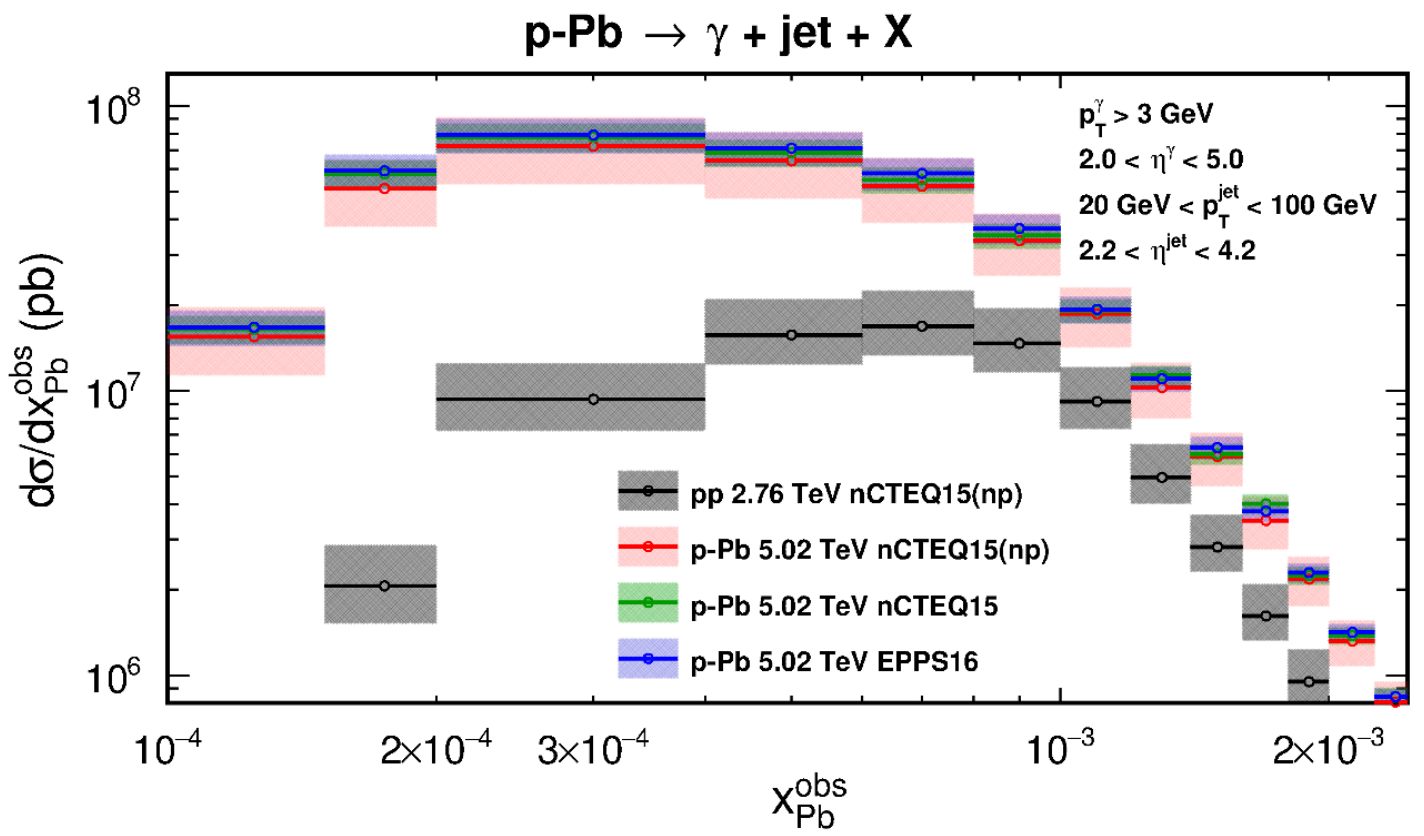

Figure 12. Nuclear PDF sensitivity of forward low- $p_{T}^{\gamma}$ photon-jet measurements with LHCb in $\mathrm{p}-\mathrm{Pb}$ collisions at the LHC with a nucleon-nucleon centre-of-mass energy of $\sqrt{s_{N N}}=5.02 \mathrm{TeV}$. Predictions with nCTEQ15(np) PDFs (red) are compared to those with nCTEQ15 including pion data from RHIC (green) and EPSS16 including weak boson and jet data from LHC (blue). The nCTEQ15 pp baseline is also shown (grey).

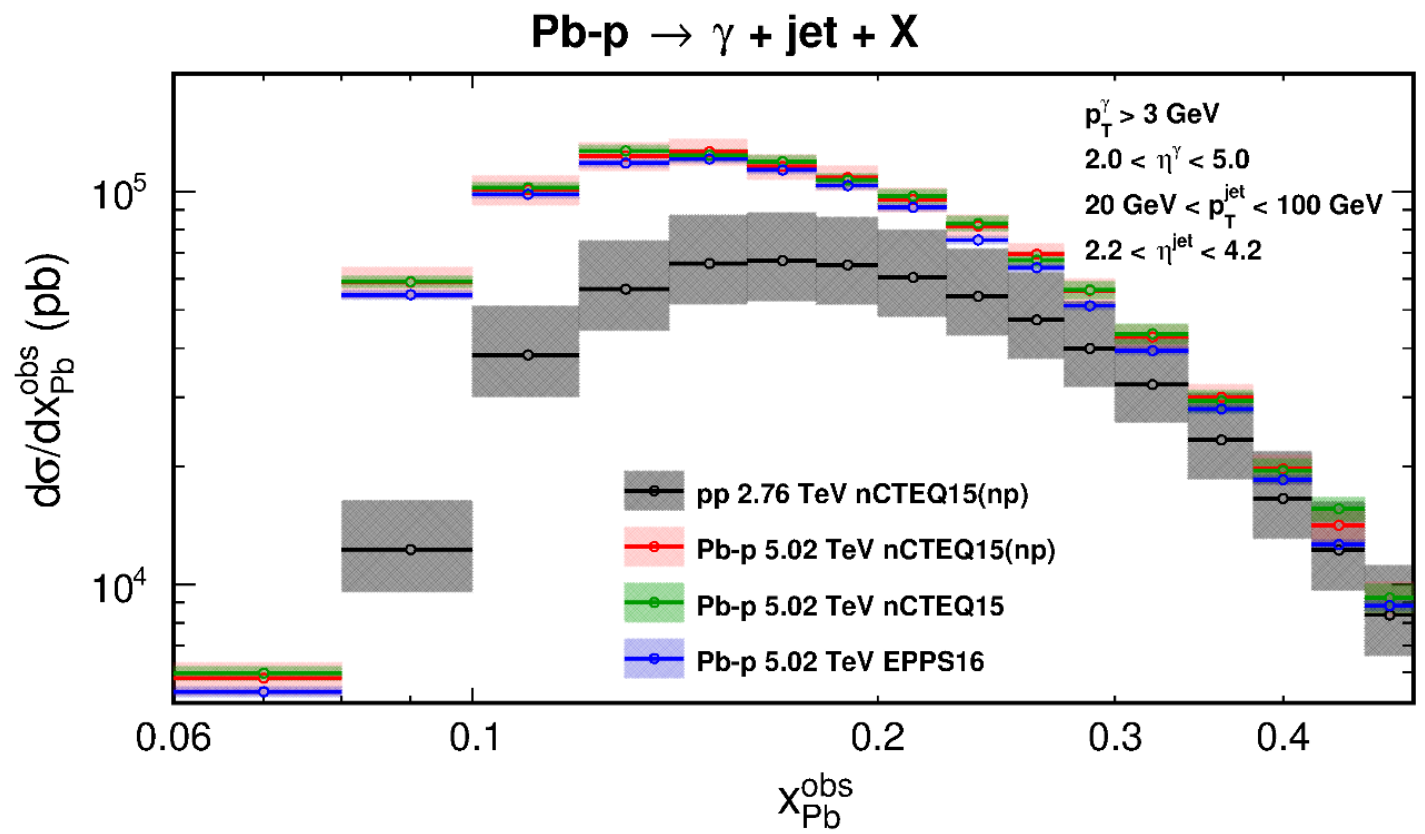

Figure 13. Same as figure 12, but for $\mathrm{Pb}-\mathrm{p}$ collisions. 
produced in association with jets as measured by $\mathrm{CMS}$ in $\mathrm{pp}$ and $\mathrm{pPb}$ collisions at various centre-of-mass energies of the LHC. For inclusive photons, we found very good agreement and, as expected, considerably reduced scale uncertainties compared to $\mathrm{LO}+\mathrm{PS}$ predictions with PYTHIA. For associated photon+jet production, the transverse momentum balance and azimuthal correlations could be described for the first time correctly at NLO.

For the extraction of the thermal photon spectrum in $\mathrm{PbPb}$ collisions, the ratio of inclusive over decay photons $R_{\gamma}$ represents an important quantity that allows to eliminate the contribution from decay photons. We predicted this quantity at NLO and NLO+PS, i.e. more reliably than it was done previously by the ALICE collaboration, and thus reduced the theoretical uncertainty considerably. The implementation of medium effects in heavyion collisions is left for future work.

We then made predictions for $\mathrm{pPb}$ collisions for ongoing analyses at $\sqrt{s_{N N}}=5.02 \mathrm{TeV}$ in all four major LHC experiments. In particular, ALICE allows to measure also low transverse momenta, whereas LHCb has very good forward instrumentation. We studied the potential of these analyses for future determinations of nuclear PDFs in distributions in the observed parton momentum fraction in the lead ion $x_{\mathrm{Pb}}^{\mathrm{obs}}$. We found that $\mathrm{LHC}$ photon data could have an important impact on the determination of nuclear effects such as shadowing at low $x$.

ATLAS has also measured isolated prompt photon production in $\mathrm{PbPb}$ collisions at a nucleon-nucleon centre-of-mass energy of $\sqrt{s_{N N}}=2.76 \mathrm{TeV}$ [13]. After scaling the data by the mean nuclear thickness $\left\langle T_{A A}\right\rangle$, they agree with NLO JETPHOX predictions in all rapidity and centrality regions within statistical and systematic uncertainties. This supports the interpretation that the strong suppression observed for the production of jets and hadrons in $\mathrm{PbPb}$ collisions compared to the scaled pp measurement is due to a strong partonic energy loss in the medium. We expect this conclusion to hold also for our similar NLO+PS POWHEG+PYTHIA results. A consistent matching of our NLO calculations with a medium-modified PS is, however, beyond the scope of the present work.

\section{Acknowledgments}

We thank M. Cacciari, D. d'Enterria and Yen-Jie Lee for useful discussions. This work has been supported by the BMBF under contract 05H15PMCCA and by the DFG through the Research Training Network 2149 "Strong and weak interactions - from hadrons to dark matter".

Open Access. This article is distributed under the terms of the Creative Commons Attribution License (CC-BY 4.0), which permits any use, distribution and reproduction in any medium, provided the original author(s) and source are credited.

\section{References}

[1] S. Albino, M. Klasen and S. Soldner-Rembold, Strong coupling constant from the photon structure function, Phys. Rev. Lett. 89 (2002) 122004 [hep-ph/0205069] [INSPIRE]. 
[2] M. Klasen, Theory of hard photoproduction, Rev. Mod. Phys. 74 (2002) 1221 [hep-ph/0206169] [INSPIRE].

[3] T. Stavreva et al., Probing gluon and heavy-quark nuclear PDFs with $\gamma+Q$ production in $p A$ collisions, JHEP 01 (2011) 152 [arXiv: 1012.1178] [INSPIRE].

[4] M. Brandt, M. Klasen and F. König, Nuclear parton density modifications from low-mass lepton pair production at the LHC, Nucl. Phys. A 927 (2014) 78 [arXiv:1401.6817] [INSPIRE].

[5] M. Klasen, K. Kovarik and J. Potthoff, Nuclear parton density functions from jet production in DIS at an EIC, Phys. Rev. D 95 (2017) 094013 [arXiv:1703.02864] [INSPIRE].

[6] P.B. Arnold, G.D. Moore and L.G. Yaffe, Photon emission from quark gluon plasma: Complete leading order results, JHEP 12 (2001) 009 [hep-ph/0111107] [INSPIRE].

[7] M. Klasen, C. Klein-Bösing, F. König and J.P. Wessels, How robust is a thermal photon interpretation of the ALICE low- $p_{T}$ data?, JHEP 10 (2013) 119 [arXiv:1307.7034] [INSPIRE].

[8] X.-N. Wang, Z. Huang and I. Sarcevic, Jet quenching in the opposite direction of a tagged photon in high-energy heavy ion collisions, Phys. Rev. Lett. 77 (1996) 231 [hep-ph/9605213] [INSPIRE].

[9] T. Sjöstrand, S. Mrenna and P.Z. Skands, A brief introduction to PYTHIA 8.1, Comput. Phys. Commun. 178 (2008) 852 [arXiv:0710.3820] [InSPIRE].

[10] ALICE collaboration, Inclusive photon production at forward rapidities in proton-proton collisions at $\sqrt{s}=$ 0.9, 2.76 and $7 \mathrm{TeV}$, Eur. Phys. J. C 75 (2015) 146 [arXiv:1411.4981] [INSPIRE].

[11] ALICE collaboration, Direct photon production in $\mathrm{Pb}-\mathrm{Pb}$ collisions at $\sqrt{s_{\mathrm{NN}}}=2.76 \mathrm{TeV}$, Phys. Lett. B 754 (2016) 235 [arXiv: 1509.07324] [INSPIRE].

[12] ATLAS collaboration, Measurement of the cross section for inclusive isolated-photon production in pp collisions at $\sqrt{s}=13 \mathrm{TeV}$ using the ATLAS detector, Phys. Lett. B 770 (2017) 473 [arXiv: 1701.06882] [INSPIRE].

[13] ATLAS collaboration, Centrality, rapidity and transverse momentum dependence of isolated prompt photon production in lead-lead collisions at $\sqrt{s_{\mathrm{NN}}}=2.76 \mathrm{TeV}$ measured with the ATLAS detector, Phys. Rev. C 93 (2016) 034914 [arXiv:1506.08552] [InSPIRE].

[14] CMS collaboration, Study of isolated photon jet correlation in $\mathrm{PbPb}$ and pp collisions at 2.76 TeV and $\mathrm{pPb}$ collisions at 5.02 TeV, CMS-PAS-HIN-13-006 (2013).

[15] LHCb collaboration, First experimental study of photon polarization in radiative $B_{s}^{0}$ decays, Phys. Rev. Lett. 118 (2017) 021801 [arXiv: 1609.02032] [INSPIRE].

[16] S. Catani, M. Fontannaz, J.P. Guillet and E. Pilon, Cross-section of isolated prompt photons in hadron hadron collisions, JHEP 05 (2002) 028 [hep-ph/0204023] [INSPIRE].

[17] P. Aurenche, M. Fontannaz, J.-P. Guillet, E. Pilon and M. Werlen, A new critical study of photon production in hadronic collisions, Phys. Rev. D 73 (2006) 094007 [hep-ph/0602133] [INSPIRE].

[18] CDF collaboration, T. Aaltonen et al., Measurement of the inclusive isolated prompt photon cross section in pp collisions at $\sqrt{s}=1.96 \mathrm{TeV}$ using the CDF detector, Phys. Rev. D 80 (2009) 111106 [arXiv:0910.3623] [INSPIRE]. 
[19] D0 collaboration, V.M. Abazov et al., Measurement of the differential cross sections for

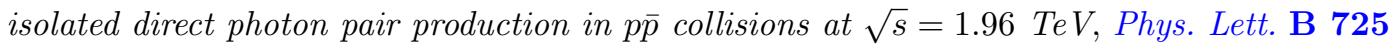
(2013) 6 [arXiv:1301.4536] [INSPIRE].

[20] D0 collaboration, V.M. Abazov et al., Measurement of the differential cross section of photon plus jet production in p p collisions at $\sqrt{s}=1.96$ TeV, Phys. Rev. D 88 (2013) 072008 [arXiv: 1308.2708] [INSPIRE].

[21] CDF collaboration, T. Aaltonen et al., Measurement of the cross section for direct-photon

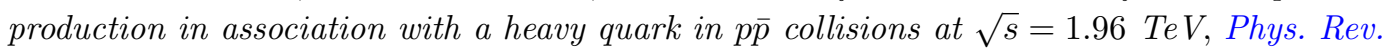
Lett. 111 (2013) 042003 [arXiv: 1303.6136] [INSPIRE].

[22] D0 collaboration, V.M. Abazov et al., Measurement of the differential photon + c-jet cross section and the ratio of differential photon $+c$ and photon $+b$ cross sections in proton-antiproton collisions at $\sqrt{s}=1.96 \mathrm{TeV}$, Phys. Lett. B 719 (2013) 354 [arXiv:1210.5033] [INSPIRE].

[23] L. Bourhis, M. Fontannaz and J.P. Guillet, Quarks and gluon fragmentation functions into photons, Eur. Phys. J. C 2 (1998) 529 [hep-ph/9704447] [INSPIRE].

[24] M. Klasen and F. König, New information on photon fragmentation functions, Eur. Phys. J. C 74 (2014) 3009 [arXiv: 1403.2290] [INSPIRE].

[25] T. Kaufmann, A. Mukherjee and W. Vogelsang, Access to photon fragmentation functions in hadronic jet production, Phys. Rev. D 93 (2016) 114021 [arXiv: 1604.07175] [INSPIRE].

[26] J.M. Campbell, R.K. Ellis, Y. Li and C. Williams, Predictions for diphoton production at the LHC through NNLO in QCD, JHEP 07 (2016) 148 [arXiv: 1603.02663] [INSPIRE].

[27] R. Boughezal et al., Color singlet production at NNLO in MCFM, Eur. Phys. J. C 77 (2017) 7 [arXiv: 1605. 08011] [INSPIRE].

[28] J.M. Campbell, T. Neumann and C. Williams, $Z \gamma$ production at NNLO including anomalous couplings, JHEP 11 (2017) 150 [arXiv:1708.02925] [INSPIRE].

[29] J.M. Campbell, H.B. Hartanto and C. Williams, Next-to-leading order predictions for $Z \gamma+j e t$ and $Z \Gamma \gamma$ final states at the LHC, JHEP 11 (2012) 162 [arXiv:1208.0566] [INSPIRE].

[30] S. Frixione, Z. Kunszt and A. Signer, Three jet cross-sections to next-to-leading order, Nucl. Phys. B 467 (1996) 399 [hep-ph/9512328] [INSPIRE].

[31] S. Frixione, P. Nason and C. Oleari, Matching NLO QCD computations with Parton Shower simulations: the POWHEG method, JHEP 11 (2007) 070 [arXiv: 0709. 2092] [INSPIRE].

[32] T. Jezo, M. Klasen and F. König, Prompt photon production and photon-hadron jet correlations with POWHEG, JHEP 11 (2016) 033 [arXiv: 1610.02275] [INSPIRE].

[33] S. Hoeche, S. Schumann and F. Siegert, Hard photon production and matrix-element parton-shower merging, Phys. Rev. D 81 (2010) 034026 [arXiv:0912.3501] [INSPIRE].

[34] T. Gehrmann, N. Greiner and G. Heinrich, Precise QCD predictions for the production of a photon pair in association with two jets, Phys. Rev. Lett. 111 (2013) 222002 [arXiv: 1308.3660] [INSPIRE].

[35] S. Badger, A. Guffanti and V. Yundin, Next-to-leading order QCD corrections to di-photon production in association with up to three jets at the Large Hadron Collider, JHEP 03 (2014) 122 [arXiv: 1312.5927] [INSPIRE].

[36] Z. Bern et al., Next-to-leading order $\gamma \gamma+2$-jet production at the LHC, Phys. Rev. D 90 (2014) 054004 [arXiv: 1402.4127] [INSPIRE]. 
[37] F. Siegert, A practical guide to event generation for prompt photon production with Sherpa, J. Phys. G 44 (2017) 044007 [arXiv: 1611.07226] [InSPIRE].

[38] T. Fritzsche et al., The implementation of the renormalized complex MSSM in FeynArts and FormCalc, Comput. Phys. Commun. 185 (2014) 1529 [arXiv:1309.1692] [InSPIRE].

[39] J. Alwall, M. Herquet, F. Maltoni, O. Mattelaer and T. Stelzer, MadGraph 5: going beyond, JHEP 06 (2011) 128 [arXiv:1106.0522] [INSPIRE].

[40] J.A.M. Vermaseren, New features of FORM, math-ph/0010025 [INSPIRE].

[41] T. Hahn and M. Pérez-Victoria, Automatized one loop calculations in four-dimensions and D-dimensions, Comput. Phys. Commun. 118 (1999) 153 [hep-ph/9807565] [InSPIRE].

[42] J. Alwall et al., The automated computation of tree-level and next-to-leading order differential cross sections and their matching to parton shower simulations, JHEP 07 (2014) 079 [arXiv: 1405.0301] [inSPIRE].

[43] S. Catani and M.H. Seymour, A general algorithm for calculating jet cross-sections in NLO QCD, Nucl. Phys. B 485 (1997) 291 [Erratum ibid. B 510 (1998) 503] [hep-ph/9605323] [INSPIRE].

[44] K. Hasegawa, S. Moch and P. Uwer, AutoDipole: automated generation of dipole subtraction terms, Comput. Phys. Commun. 181 (2010) 1802 [arXiv:0911.4371] [INSPIRE].

[45] L. D'Errico and P. Richardson, Next-to-leading-order Monte Carlo simulation of diphoton production in hadronic collisions, JHEP 02 (2012) 130 [arXiv:1106.3939] [INSPIRE].

[46] L. Barze et al., Wr production in hadronic collisions using the POWHEG+MiNLO method, JHEP 12 (2014) 039 [arXiv: 1408.5766] [INSPIRE].

[47] NNPDF collaboration, R.D. Ball et al., Parton distributions from high-precision collider data, Eur. Phys. J. C 77 (2017) 663 [arXiv:1706.00428] [inSPIRE].

[48] NNPDF collaboration, R.D. Ball et al., Parton distributions with QED corrections, Nucl. Phys. B 877 (2013) 290 [arXiv: 1308.0598] [INSPIRE].

[49] P. Skands, S. Carrazza and J. Rojo, Tuning PYTHIA 8.1: the Monash 2013 Tune, Eur. Phys. J. C 74 (2014) 3024 [arXiv:1404.5630] [INSPIRE].

[50] M. Cacciari and D. d'Enterria, private communication.

[51] M. Cacciari, G.P. Salam and G. Soyez, The anti-k $k_{t}$ jet clustering algorithm, JHEP 04 (2008) 063 [arXiv: 0802.1189] [INSPIRE].

[52] X.-N. Wang and M. Gyulassy, HIJING: a Monte Carlo model for multiple jet production in $p$ p, p A and A A collisions, Phys. Rev. D 44 (1991) 3501 [InSPIRE].

[53] CMS collaboration, Study of isolated-photon + jet correlations in $\mathrm{PbPb}$ and $p p$ collisions at $\sqrt{s_{N N}}=5.02 \mathrm{TeV}$, CMS-PAS-HIN-16-002 (2016).

[54] ATLAS collaboration, Measurement of the cross section for isolated-photon plus jet production in pp collisions at $\sqrt{s}=13 \mathrm{TeV}$ using the ATLAS detector, ATLAS-CONF-2017-059 (2017).

[55] K. Kovarik et al., nCTEQ15 - Global analysis of nuclear parton distributions with uncertainties in the CTEQ framework, Phys. Rev. D 93 (2016) 085037 [arXiv:1509.00792] [INSPIRE].

[56] D. Stump et al., Inclusive jet production, parton distributions and the search for new physics, JHEP 10 (2003) 046 [hep-ph/0303013] [INSPIRE]. 
[57] K.J. Eskola, P. Paakkinen, H. Paukkunen and C.A. Salgado, EPPS16: nuclear parton distributions with LHC data, Eur. Phys. J. C 77 (2017) 163 [arXiv: 1612.05741] [INSPIRE].

[58] CMS Collaboration, Production of pairs of isolated photons in association with jets in pp collisions at $\sqrt{s}=7 \mathrm{TeV}$, CMS-PAS-SMP-14-021 (2014).

[59] ALICE collaboration, M. Germain, Direct photon measurements in $p p$ and $\mathrm{Pb}-\mathrm{Pb}$ collisions with the ALICE experiment, in the proceedings of the XXVI International Conference on Ultrarelativistic Heavy-Ion Collisions (Quark Matter 2017), February 5-11, Chicago, U.S.A. (2017), Nucl. Phys. A 967 (2017) 696.

[60] ALICE collaboration, Measurement of charged jet production cross sections and nuclear modification in $p-P b$ collisions at $\sqrt{s_{\mathrm{NN}}}=5.02$ TeV, Phys. Lett. B 749 (2015) 68 [arXiv: 1503.00681] [INSPIRE].

[61] ALICE collaboration, Centrality dependence of charged jet production in $p-P b$ collisions at $\sqrt{s_{\mathrm{NN}}}=5.02 \mathrm{TeV}$, Eur. Phys. J. C 76 (2016) 271 [arXiv:1603.03402] [INSPIRE].

[62] LHCb collaboration, Measurement of forward $W$ and $Z$ boson production in association with jets in proton-proton collisions at $\sqrt{s}=8 \mathrm{TeV}$, JHEP 05 (2016) 131 [arXiv:1605.00951] [INSPIRE]. 